\title{
Molecular genomic characterization of severe fever with thrombocytopenia syndrome virus isolates from South Korea
}

\author{
Yu Jung Won ${ }^{1}$, Lae Hyung Kang ${ }^{1}$, \\ Sung Geun Lee ${ }^{2}$, Seung Won Park ${ }^{3}$, \\ Jae Ik Han ${ }^{4}$, and Soon Young Paik ${ }^{1 \star}$ \\ ${ }^{1}$ Department of Microbiology, College of Medicine, \\ The Catholic University of Korea, Seoul 06591, Republic of Korea \\ ${ }^{2}$ Korea Zoonosis Research Institute, Chonbuk National University, \\ Iksan 54596, Republic of Korea \\ ${ }^{3}$ Department of Biotechnology, Daegu Catholic University, \\ Gyeongsan 38430, Republic of Korea \\ ${ }^{4}$ Laboratory of Wildlife Diseases, College of Veterinary Medicine, \\ Chonbuk National University, Iksan 54596, Republic of Korea \\ (Received Apr 3, 2019 / Revised May 27, 2019 / Accepted Jun 17, 2019)
}

Severe fever with thrombocytopenia syndrome (SFTS) is a tick-borne emerging infectious disease caused by the SFTS virus (SFTSV) and is a threat to public health due to its high fatality rate. However, details on tick-to-human transmission of SFTSV are limited. In this study, we determined the wholegenome sequence of a South Korean SFTSV strain (CUKJJ01), compared it to those of other recent human SFTSV isolates, and identified the genetic variations and relationships among the SFTSV strains. The genome of CUK-JJ01 was consistent with the genome of other members of the genus Phlebovirus, including the large (L), medium (M), and small (S) segments of 6368,3378 , and 1744 nucleotides, respectively. Based on amino acid sequences of the $M$ and $S$ segments, which are used to distinguish the six SFTSV genotypes, CUK-JJ01 was classified as genotype B. Segment analysis revealed that the $\mathrm{L}, \mathrm{M}$, and S segments were $97.49 \%$, $\mathbf{9 7 . 1 8 \%}$, and $97.94 \%$ similar to those of KAJNH2/2013/ Korea, ZJZHSH-FDE/2012/China, and KADGH/2013/Korea, respectively. Currently, only few studies on SFTSV have been conducted in Korean population and most were limited to serological analysis. Although the present study has limitations in terms of number of sample analyzed, the findings may serve as basis to understand the transmission and spread of SFTSV, as well as for the development of diagnostic and detection methods for viral recombinants by comparing the whole genome sequence of SFTSV isolates from South Korea and that of foreign isolates.

Keywords: SFTS, SFTSV, whole-genome sequencing, Phlebovirus, South Korea, phylogeny

*For correspondence. E-mail: paik@catholic.ac.kr; Tel.: +82-2-2258-7342 Copyright (C) 2019, The Microbiological Society of Korea

\section{Introduction}

Severe fever with thrombocytopenia syndrome (SFTS) is an emerging tick-borne viral disease characterized by fever, gastrointestinal symptoms, leukopenia, and thrombocytopenia.

It was first reported in China in 2009, and subsequently in South Korea and western Japan in 2010 and 2013, respectively (Yu et al., 2011; Kim et al., 2013; Park et al., 2014; Takahashi et al., 2014). The etiological agent, SFTS virus (SFTSV), which belongs to the genus Phlebovirus of the family Phenuiviridae, is suspected to be a tick-borne virus based on evidence of its presence in four species of ticks, Haemaphysalis longicornis, Amblyomma testudinarium, Ixodes nipponensis, and Rhipicephalus microplus (Yu et al., 2011; Zhang et al., 2012; Yun et al., 2015). Furthermore, this virus has also been isolated from or detected in domesticated animals (e.g., sheep, cattle, rodents, and dogs), small mammals such as rodents and shrews, and reptiles (Zhao et al., 2012; Niu et al., 2013; Liu et al., 2014b).

SFTS is an acute infectious disease with a high mortality rate (approximately 6-30\%) that can affect people at any age (Yu et al., 2011; Ding et al., 2013; Takahashi et al., 2014; Yoshikawa et al., 2015). Hospitalized patients that presented with clinical symptoms consistent with SFTS have been frequently reported in central and northeast China since March 2010. In 2009, the SFSV was identified as the causative agent of SFTS (Xu et al., 2011; Yu et al., 2011; Liu et al., 2014a). Importantly, human-to-human transmission of SFTSV was documented in a family cluster in China in 2011 (Bao et al., 2011). The evidence demonstrates that SFTS has become an increasing threat to public health.

Like other phleboviruses, SFTSV contains a tripartite RNA genome consisting of three single-stranded RNA segments of negative polarity, designated as the large (L), medium (M), and small (S) segments (Yu et al., 2011; Liu et al., 2014a). The genome encodes an RNA-dependent RNA polymerase (RdRp) from the L segment, viral envelope glycoproteins $\mathrm{Gn}$ and $\mathrm{Gc}$ from the $\mathrm{M}$ segment, and a nucleoprotein (NP) and nonstructural protein (NS) in an ambisense orientation from the S segment (Yu et al., 2011; Liu et al., 2014a). The nomenclature for SFTSV genotypes is based on the country of origin, such as $\mathrm{C} 1$ to $\mathrm{C} 5$ for the Chinese lineage and J1 to J3 for the Japanese lineage. The phylogenetic analysis of a viral genome is indispensable for understanding the features of genotypic variation and the relationship of strains with their geographic distribution. For instance, it has been shown that eight strains of SFTSV recovered from patients in Japan clustered to an independent lineage separate from Chinese strains (Takahashi et al., 2014). Recently, based on full-length sequencing of 
multiple SFTSV strains $(n=159)$ from China, South Korea, and Japan, Fu et al. (2016) have proposed a new nomenclature for SFTSV strains, which divides the viruses into six clades referred to as genotypes A to $\mathrm{F}$.

Since the first reported fatal case in 2012 in Gangwon Province, South Korea (Kim et al., 2013), concern regarding SFTSV has grown as the number of SFTS patients has increased annually with 36 cases reported in 2013, 55 cases in
2014, 79 cases in 2015, 165 cases in 2016, and 272 cases in 2017 (Korea Center for Disease Control, 2018). The mean mortality rate of these cases was approximately $20.9 \%$.

According to a recent study in Vietnam (Tran et al., 2019), virus-bearing Amblyomma testudinarium ticks and migratory birds played a role in dispersing SFTSV to Vietnam. In the current study, we analyzed the whole-genome sequence of a human SFTSV strain isolated from a patient who died

Table 1. Newly designed primers used in this study

\begin{tabular}{|c|c|c|c|}
\hline Primer & Sequence $\left(5^{\prime}-3^{\prime}\right)$ & Location $^{\mathrm{a}}$ & Size (bp) \\
\hline L-GSP1 & GCT GTC CAG TCA GGA TCA & $5424-5441$ & \\
\hline L-GSP2 & CAA GTA AGT ACT ATC ATC TAA & $6033-6053$ & \\
\hline L-Nested GSP & CCC TCG AGA CAG TCC CTA AA & $5771-5790$ & \\
\hline L-F1 & ACA CAG AGA CGC CCA GAT GA & $1-20$ & \multirow{2}{*}{656} \\
\hline L-R1 & GCT CAA TGT CTG CAT CCA TAG A & $635-656$ & \\
\hline $\mathrm{L}-\mathrm{F} 2$ & CAG GAT GAG GCA GAG GAG CT & $569-588$ & \multirow{2}{*}{760} \\
\hline L-R2 & CTG CCA TTC TCT TCC ACT TCT TC & $1306-1328$ & \\
\hline L-F3 & GTG GAG GGG AAG AAG TGG AAG & $1298-1318$ & \multirow{2}{*}{676} \\
\hline L-R3 & GAC TGA TCA GGA AGG TCA GGC & $1953-1973$ & \\
\hline L-F4 & GGC TCC ATG GCT GGT TCG AA & $1903-1922$ & \multirow{2}{*}{634} \\
\hline L-R4 & CTT GGT CCC AAT CTC TCT CAG & $2516-2536$ & \\
\hline L-F5 & GGA AGC AGA ACC TTG AGG AGC & $2490-2510$ & \multirow{2}{*}{696} \\
\hline L-R5 & GGA AGG CRT CTG TCA TTG CTT C & $3164-3185$ & \\
\hline L-F6 & CTG AGT CTA GGT CAT CTG ATC C & $3135-3156$ & \multirow{2}{*}{702} \\
\hline L-R6 & CTA GGG CTG GAT CTG GGT CT & $3817-3836$ & \\
\hline L-F7 & CTR CAC CAC TAC ATG CTT CTA GG & $3746-3769$ & \multirow{2}{*}{711} \\
\hline L-R7 & CTT CCT CAA GCA ATG TGC ATA C & $4436-4457$ & \\
\hline L-F8 & GAG CGA ATG CTC TTC CCT CAG & $4397-4417$ & \multirow{2}{*}{704} \\
\hline L-R8 & GCC CTC TCA ATC TGG TCT AGA A & $5079-5100$ & \\
\hline L-F9 & CCW CAG CCA TCA GAG GTC AT & $4994-5013$ & \multirow{2}{*}{675} \\
\hline L-R9 & GAT CCA GCT GCA GGA TGG YTC & $5648-5668$ & \\
\hline L-F10 & GCA TAC CTC TGG AGC AAT CGA & $5603-5623$ & \multirow{2}{*}{766} \\
\hline L-R10 & ACA CAA AGA CCG CCC AGA TCT TA & $6346-6368$ & \\
\hline M-GSP1 & CTA CTC TGC CAT ACC CAC & $2577-2594$ & \\
\hline M-GSP2 & TTC TTC TTA ATC ATG TCT TTG T & $3169-3190$ & \\
\hline M-Nested GSP & AGC TAT GAA TCC CCT CCA GGA CAC & $2870-2893$ & \\
\hline M-F1 & ACA CAG AGA CGG CCA ACA ATG ATG A & $1-25$ & \multirow{2}{*}{775} \\
\hline M-R1 & ATG AGC TCT CCT GTA CCC ATT TGG T & $751-775$ & \\
\hline M-F2 & GAG AGT CTA CCT CAG CCC TTT GAT G & $673-697$ & \multirow{2}{*}{758} \\
\hline M-R2 & GTG AGT AGC ATG AGG CCT GCA TAT C & $1406-1430$ & \\
\hline M-F3 & AGT GGA CTG CAC ATT CTG TCG TGA G & $1314-1338$ & \multirow{2}{*}{780} \\
\hline M-R3 & CCA CAT CCA TCA GAG CAG CCA CT & $2071-2093$ & \\
\hline M-F4 & ATC AGT GAG GAG ATG TCG TTG GGC & $1953-1976$ & \multirow{2}{*}{685} \\
\hline M-R4 & CTG GCT GTC ATG GCT TCT CTC AAA C & $2613-2637$ & \\
\hline M-F5 & AGA GCT ACG AGT GGA TGA CGC TG & $2514-2536$ & \multirow{2}{*}{865} \\
\hline M-R5 & ACA CAA AGA CCG GCC AAC ACT TC & $3356-3378$ & \\
\hline S-GSP1 & TGA TGA AGA GCT GAG AAC & $826-843$ & \\
\hline S-GSP2 & TTC TGC CCT CTC AAC CAG & $1412-1429$ & \\
\hline S-Nested GSP & AGGGATCCTCTCCATGC & $1095-1111$ & \\
\hline S-F1 & ACA CAA AGA CCC CCT TCA TTT G & $1-23$ & \multirow{2}{*}{615} \\
\hline S-R1 & CCT CTT AGC CTT GCA AGT GAC & $595-615$ & \\
\hline S-F2 & GCG AAG RCT TAG ACT TGG GT & $514-533$ & \multirow{2}{*}{651} \\
\hline S-R2 & CTA TCA ATG TGA AGA TGC GYG & $1144-1164$ & \\
\hline S-F3 & GAA GAC AGA GTT CAC AGC AGC & $1076-1096$ & \multirow{2}{*}{669} \\
\hline S-R3 & ACA CAA AGA CCC CCA AAA AAG G & $1723-1744$ & \\
\hline $3^{\prime}$-Oligo (dT)-anchor-R & GAG GAC TCG AGC TCA AGC TTT TTT TTT TTT TTT & $3^{\prime}$-end poly A tail & \\
\hline $3^{\prime}$-Anchor-R & GAG GAC TCG AGC TCA AGC & & \\
\hline
\end{tabular}


from SFTS in Jeju Island, South Korea in October 2017 and compared it to those of other human SFTSV strains to determine the genetic and evolutionary characteristics of this important pathogen. We expect that these data will prove useful, not only for advancing research in the molecular biology of SFTSV, but also for basic epidemiological analyses and tracking the international spread of the virus.

\section{Materials and Methods}

\section{Ethics statement}

Jeju Halla General Hospital received written consent for sample collection from each patient with SFTSV infection. All participants were adults, and the study was approved by the institutional review board (IRB) of the Catholic Medical Center Office of Human Research Protection Program (CMC OHRP) of South Korea (approval no. MC18SESI0077). The authors assert that all procedures contributing to this work comply with the ethical standards of the relevant national and institutional committees on human experimentation and with the Helsinki Declaration of 1975, as revised in 2008.

\section{Virus propagation, cell lines, and viral RNA extraction}

For propagation, the virus was passaged five times on confluent monolayers of Vero cells (KCLB 10081; Korean Cell Line Bank) in Dulbecco's Modified Eagle Medium (DMEM; Hyclone) containing 10\% fetal bovine serum (FBS; Hyclone) with penicillin $(100 \mathrm{IU} / \mathrm{ml}) /$ streptomycin $(100 \mu \mathrm{g} / \mathrm{ml} ; \mathrm{P} / \mathrm{S}$, Hyclone) in a $37^{\circ} \mathrm{C}$ incubator supplemented with $5 \% \mathrm{CO}_{2}$. Cell culture supernatants were collected after seven days and stored at $-80^{\circ} \mathrm{C}$ as working virus stocks for whole-genome sequencing. Viral RNA was extracted from $140 \mu \mathrm{l}$ of the viral stocks using QIAamp Viral RNA Mini Kits (Qiagen) according to the manufacturer's instructions. Single stranded complementary DNA (cDNA) was synthesized from the viral RNA as template using primers specific for each segment and a cDNA Synthesis Kit (TaKaRa Bio) according to the manufacturer's instructions.

\section{Whole-genome sequencing}

For whole-genome sequencing, one to six overlapping PCR fragments covering the SFTSV genome, including full-length $\mathrm{L}, \mathrm{M}$, and $\mathrm{S}$ segments, were amplified by polymerase chain reaction (PCR) using the cDNA as template and Taq DNA polymerase (Qiagen) on an S1000 thermal cycler (Bio-Rad). The PCR primers were designed based on the full-genome sequence of the detected SFTSV (Table 1). PCR amplification of each segment was performed using a profile of $94^{\circ} \mathrm{C}$ for $3 \mathrm{~min}$, followed by $35 \mathrm{cycles}$ of $94^{\circ} \mathrm{C}$ for $30 \mathrm{sec}$, annealing for $30 \mathrm{sec}$ at 53 or $60^{\circ} \mathrm{C}$ according to the primer sets for each segment, and $72^{\circ} \mathrm{C}$ for $1 \mathrm{~min}$, followed by a final extension at $72^{\circ} \mathrm{C}$ for $10 \mathrm{~min}$. All PCR products were examined by electrophoresis on $2 \%$ agarose gels stained with ethidium bromide.

Determination of the $5^{\prime}$ - and $3^{\prime}$-ends of the SFTSV genomic RNA

To determine the $5^{\prime}$-ends of the SFTSV genomic RNA, rapid amplification of cDNA ends (RACE) was performed using the $5^{\prime}$ RACE System for Rapid Amplification of cDNA Ends Version 2.0 Kit (Invitrogen) according to the manufacturer's recommendations. Three primers, GSP1, GSP2, and nested GSP, were designed for $5^{\prime}$ RACE PCR based on the L sequence (Table 1). To obtain the exact sequence of the $3^{\prime}$-end of the SFTSV genomic RNA, cDNA was synthesized by reverse transcription using $3^{\prime}$-oligo (dT)-anchor-R (Table 1). The second PCR was conducted using the S-F and 3 '-anchor$\mathrm{R}$ primers (Table 1) under the following conditions: $30 \mathrm{cy}-$ cles of $94^{\circ} \mathrm{C}$ for $30 \mathrm{sec}, 55^{\circ} \mathrm{C}$ for $30 \mathrm{sec}$, and $72^{\circ} \mathrm{C}$ for $1 \mathrm{~min}$ followed by $72^{\circ} \mathrm{C}$ for $10 \mathrm{~min}$.

\section{Cloning and sequencing of the complete genome}

All PCR products were extracted using a HiYield Gel/PCR DNA Fragments Extraction Kit (RBC Bioscience) and cloned into pGEM-T Easy Vectors (Promega). The cloned vectors were transformed into Escherichia coli DH5a competent cells (RBC Bioscience) according to the manufacturer's instructions and selected at $37^{\circ} \mathrm{C}$ for $16-18 \mathrm{~h}$ on Luria-Bertani agar plates (Duchefa) containing $40 \mathrm{mg} / \mathrm{ml} \mathrm{X-gal,} 0.1 \mathrm{mM}$ isopropyl- $\beta$-d-thiogalactoside, and $50 \mathrm{mg} / \mathrm{ml}$ ampicillin. Selected clones were inoculated into Luria-Bertani broth (Duchefa) and incubated overnight in a shaking incubator (IS$971 \mathrm{R}$, Jeiotech) at $37^{\circ} \mathrm{C}$ and $200 \mathrm{rpm}$. Plasmid DNA was purified using a HiYield Plasmid Mini Kit (RBC Bioscience) and sequenced (Macrogen). The sequencing results were analyzed using the Basic Local Alignment Search Tool (BLAST) provided by the National Center for Biotechnology Information (NCBI; National Institutes of Health).

\section{Phylogenetic analysis}

Comparative sequence analyses, including sequence alignments and estimation of genetic distances, were performed using the ClustalW program of the Molecular Evolutionary Genetic Analysis (MEGA) software, version 7.0 (Tamura et al., 2013). Phylogenetic trees were constructed using the neighbor-joining method with a Kimura two-parameter model provided in the MEGA software (Saitou and Nei, 1987) and branch support was calculated based on 1,000 bootstrap replicates. The complete-genome sequences and partial-genome sequences were obtained from the NCBI database.

\section{Results}

\section{Nucleotide sequence identities}

The genome of the human-derived South Korean SFTSV strain CUK-JJ01 (GenBank no. MH937372-MH937374) analyzed in this study consisted of L, M, and S segments of 6368,3378 , and 1744 nucleotides (nt), respectively. The L segment had a 6255-nt long open reading frame (ORF) for the RNA-dependent RNA polymerase gene that encoded a predicted 2084 amino acid (aa) protein. The M segment contained an ORF for a 3222-nt precursor of the glycoprotein gene encoding for proteins $\mathrm{Gn}$ (516 aa) and Gc (511 aa). The $\mathrm{S}$ segment contained 882- and 738-nt long ORFs, which encoded the NS protein (293 aa) and NP (245 aa), respectively. The non-coding regions (NCRs) of the $\mathrm{L}, \mathrm{M}$, and S segments 
at the $5^{\prime}$-termini were 16-, 18-, and 42-nt long, respectively, and at the $3{ }^{\prime}$-termini were 97-, 138-, and 29-nt long, respectively. Segment analysis of CUK-JJ01 revealed that the L segment was $97.49 \%$ similar to that of KAJNH2/2013/Korea (GenBank accession no. KU507547), the M segment was 97.18\% similar to that of ZJZHSH-FDE/2012/China (GenBank accession no. KR017859), and the S segment was $97.94 \%$ similar to that of KADGH/2013/Korea (GenBank accession no. KU507553). Moreover, NP-encoding region of the S segment of strain CUK-JJ01 demonstrated $98.24 \%$ sequence identity at the nt level with the genotype D strain KP663731
(KASJH). Surprisingly, sequence comparison of the NP ORFs of the S segment indicated that CUK-JJ01 was most closely related to SFTSV genotype B reference strains at 95.25-98.64\% identity (Table 2). The highest sequence identity (98.64\%) was observed with the reference strain KU507543 (KADGH), which was isolated in South Korea. A comparative analysis of the CUK-JJ01 nt sequence of the NS ORF of the S segment with that of the SFTSV reference sequences indicated that CUK-JJ01 was most closely related to the South Korean reference strain KU507543 (KADGH) at $97.28 \%$ identity (Table 2).

Table 2. Nucleotide sequence identities of the full-length genome and L, M, and S-NP/NSs genes between the SFTSV CUK-JJ01 strain and reference strains

\begin{tabular}{|c|c|c|c|c|c|c|c|c|}
\hline \multirow{2}{*}{ Reference strain } & \multirow{2}{*}{ Accession number } & \multirow{2}{*}{ Genotype } & \multirow{2}{*}{ Collection date } & \multirow{2}{*}{ Country } & \multicolumn{4}{|c|}{ Nucleotide sequence identity (\%) } \\
\hline & & & & & $\mathrm{L}$ & M & S-NP & S-NSs \\
\hline AH15 & HQ141592 & $\mathrm{A}$ & 2010 & China & $95.98 \%$ & $93.82 \%$ & $95.79 \%$ & $94.67 \%$ \\
\hline CB2 & KY789434 & A & 2015 & Korea & $96.11 \%$ & $93.92 \%$ & $95.79 \%$ & $95.35 \%$ \\
\hline HL_Adult_G2 & KF791962 & $\mathrm{A}$ & 2013 & China & $95.97 \%$ & $93.85 \%$ & $95.12 \%$ & $94.78 \%$ \\
\hline HL_Egg_G2 & KF791959 & A & 2013 & China & $95.97 \%$ & $93.92 \%$ & $95.12 \%$ & $94.78 \%$ \\
\hline HL_Injected & KF791958 & A & 2013 & China & $95.97 \%$ & $93.92 \%$ & $95.12 \%$ & $94.78 \%$ \\
\hline HL_Larvae_G2 & KF791960 & A & 2013 & China & $95.97 \%$ & $93.85 \%$ & $95.12 \%$ & $94.78 \%$ \\
\hline HL_Nymph_G2 & KF791961 & A & 2013 & China & $95.97 \%$ & $93.85 \%$ & $95.12 \%$ & $94.78 \%$ \\
\hline JS3 & HQ141601 & A & 2010 & China & $96.00 \%$ & $93.85 \%$ & $95.52 \%$ & $95.01 \%$ \\
\hline LN3 & HQ141610 & A & 2010 & China & $96.11 \%$ & $93.89 \%$ & $94.57 \%$ & $95.46 \%$ \\
\hline SPL087A & AB983515 & A & 2013 & Japan & $95.79 \%$ & $93.70 \%$ & $94.84 \%$ & $94.67 \%$ \\
\hline 01_Zhejiang & KJ597825 & B & 2011 & China & $97.31 \%$ & $97.11 \%$ & $97.96 \%$ & $96.94 \%$ \\
\hline CB1 & KY789433 & B & 2014 & Korea & $97.12 \%$ & $96.46 \%$ & $95.79 \%$ & $95.80 \%$ \\
\hline CB3 & KY789435 & B & 2016 & Korea & $96.24 \%$ & $95.81 \%$ & $95.93 \%$ & $95.69 \%$ \\
\hline KACNH3 & КР663743 & B & 2014 & Korea & $96.08 \%$ & $95.56 \%$ & $96.74 \%$ & $95.57 \%$ \\
\hline KADGH & KU507543 & B & 2013 & Korea & $97.20 \%$ & $96.46 \%$ & $98.64 \%$ & $97.28 \%$ \\
\hline KAGBH5 & KР663737 & B & 2014 & Korea & $96.37 \%$ & $95.90 \%$ & $96.20 \%$ & $96.94 \%$ \\
\hline KAGBH6 & KР663740 & B & 2014 & Korea & $96.42 \%$ & $95.59 \%$ & $95.25 \%$ & $96.94 \%$ \\
\hline KAGNH & KU507545 & B & 2013 & Korea & $96.19 \%$ & $95.28 \%$ & $96.07 \%$ & $96.37 \%$ \\
\hline KAGNH4 & KU507546 & $\mathrm{B}$ & 2013 & Korea & $96.22 \%$ & $95.65 \%$ & $97.15 \%$ & $97.28 \%$ \\
\hline KAGWT & KY273136 & B & 2013 & Korea & $96.42 \%$ & $95.62 \%$ & $96.20 \%$ & $96.48 \%$ \\
\hline KAJNH2 & KU507547 & B & 2013 & Korea & $97.49 \%$ & $96.87 \%$ & $96.07 \%$ & $96.37 \%$ \\
\hline Zhao & KF374682 & $\mathrm{B}$ & 2013 & China & $97.38 \%$ & $97.14 \%$ & $97.83 \%$ & $96.82 \%$ \\
\hline ZJZHSH-FDE & KR017840 & B & 2012 & China & $97.39 \%$ & $97.18 \%$ & $97.96 \%$ & $96.82 \%$ \\
\hline ZJZHSH-LWL & KR017844 & B & 2014 & China & $97.30 \%$ & $97.05 \%$ & $97.96 \%$ & $96.94 \%$ \\
\hline ZJZHSH-WRF & KR017845 & B & 2014 & China & $97.31 \%$ & $97.14 \%$ & $97.83 \%$ & $96.59 \%$ \\
\hline 2011YPQ17 & KF711887 & $\mathrm{D}$ & 2011 & China & $95.47 \%$ & $93.51 \%$ & $95.12 \%$ & $94.55 \%$ \\
\hline AHL & JQ670934 & $\mathrm{D}$ & 2011 & China & $95.58 \%$ & $94.29 \%$ & $95.66 \%$ & $94.67 \%$ \\
\hline HB154 & JQ733561 & $\mathrm{D}$ & 2011 & China & $95.60 \%$ & $93.48 \%$ & $94.71 \%$ & $95.01 \%$ \\
\hline HB155 & JQ733564 & $\mathrm{D}$ & 2011 & China & $95.54 \%$ & $93.45 \%$ & $94.84 \%$ & $94.78 \%$ \\
\hline HB156 & JQ733567 & $\mathrm{D}$ & 2011 & China & $95.55 \%$ & $93.58 \%$ & $94.71 \%$ & $95.01 \%$ \\
\hline KASJH & КР663731 & $\mathrm{D}$ & 2014 & Korea & $95.79 \%$ & $93.67 \%$ & $98.24 \%$ & $96.71 \%$ \\
\hline $\mathrm{SD} 24$ & HM802200 & $\mathrm{D}$ & 2010 & China & $95.58 \%$ & $93.58 \%$ & $94.57 \%$ & $94.55 \%$ \\
\hline JS4 & HQ141604 & $\mathrm{E}$ & 2010 & China & $95.71 \%$ & $94.17 \%$ & $96.20 \%$ & $95.12 \%$ \\
\hline SD4 & HM802202 & $\mathrm{E}$ & 2010 & China & $95.74 \%$ & $94.01 \%$ & $94.84 \%$ & $95.12 \%$ \\
\hline AH12 & HQ116417 & $\mathrm{F}$ & 2010 & China & $96.08 \%$ & $94.01 \%$ & $94.84 \%$ & $94.67 \%$ \\
\hline AH-YTY_ & KR017830 & $\mathrm{F}$ & 2012 & China & $95.97 \%$ & $94.04 \%$ & $94.84 \%$ & $94.67 \%$ \\
\hline $\mathrm{AHZ}$ & JQ670929 & $\mathrm{F}$ & 2011 & China & $95.92 \%$ & $93.76 \%$ & $94.57 \%$ & $95.12 \%$ \\
\hline Gangwon & KF358691 & $\mathrm{F}$ & 2012 & Korea & $96.03 \%$ & $94.01 \%$ & $94.84 \%$ & $94.55 \%$ \\
\hline HN6 & HQ141595 & $\mathrm{F}$ & 2010 & China & $95.86 \%$ & $93.82 \%$ & $94.57 \%$ & $94.67 \%$ \\
\hline JS2012-tick01 & KC473540 & $\mathrm{F}$ & 2012 & China & $95.84 \%$ & $93.73 \%$ & $94.71 \%$ & $95.12 \%$ \\
\hline LN2 & HQ141607 & $\mathrm{F}$ & 2010 & China & $95.92 \%$ & $93.79 \%$ & $95.25 \%$ & $94.67 \%$ \\
\hline QD7 & KR706567 & $\mathrm{F}$ & 2014 & China & $95.76 \%$ & $93.64 \%$ & $95.39 \%$ & $95.35 \%$ \\
\hline SDLZtick12 & JQ684871 & $\mathrm{F}$ & 2012 & China & $95.86 \%$ & $93.82 \%$ & $96.20 \%$ & $94.78 \%$ \\
\hline
\end{tabular}

SFTSV, severe fever with thrombocytopenia syndrome virus. 
Genotype

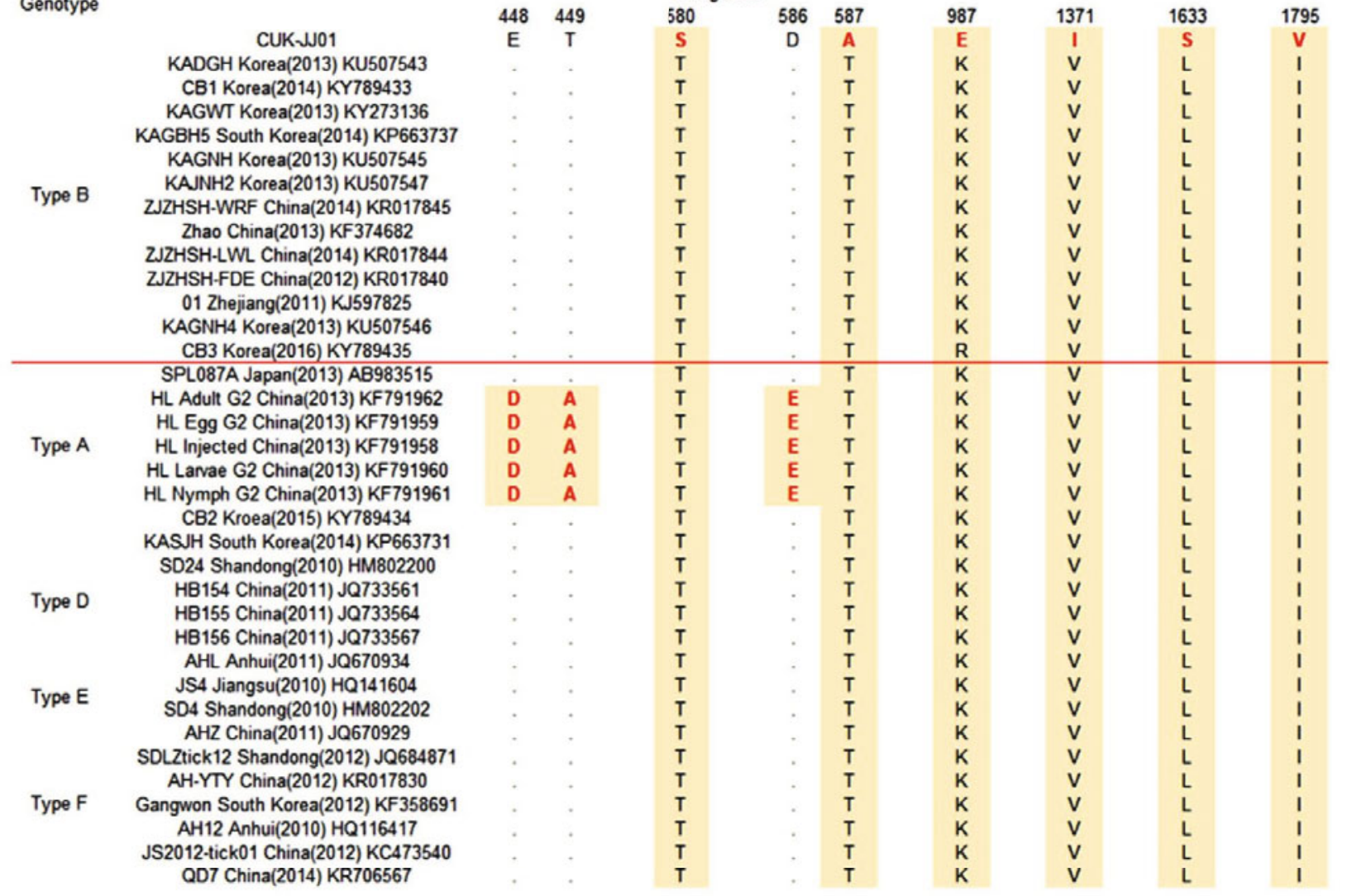

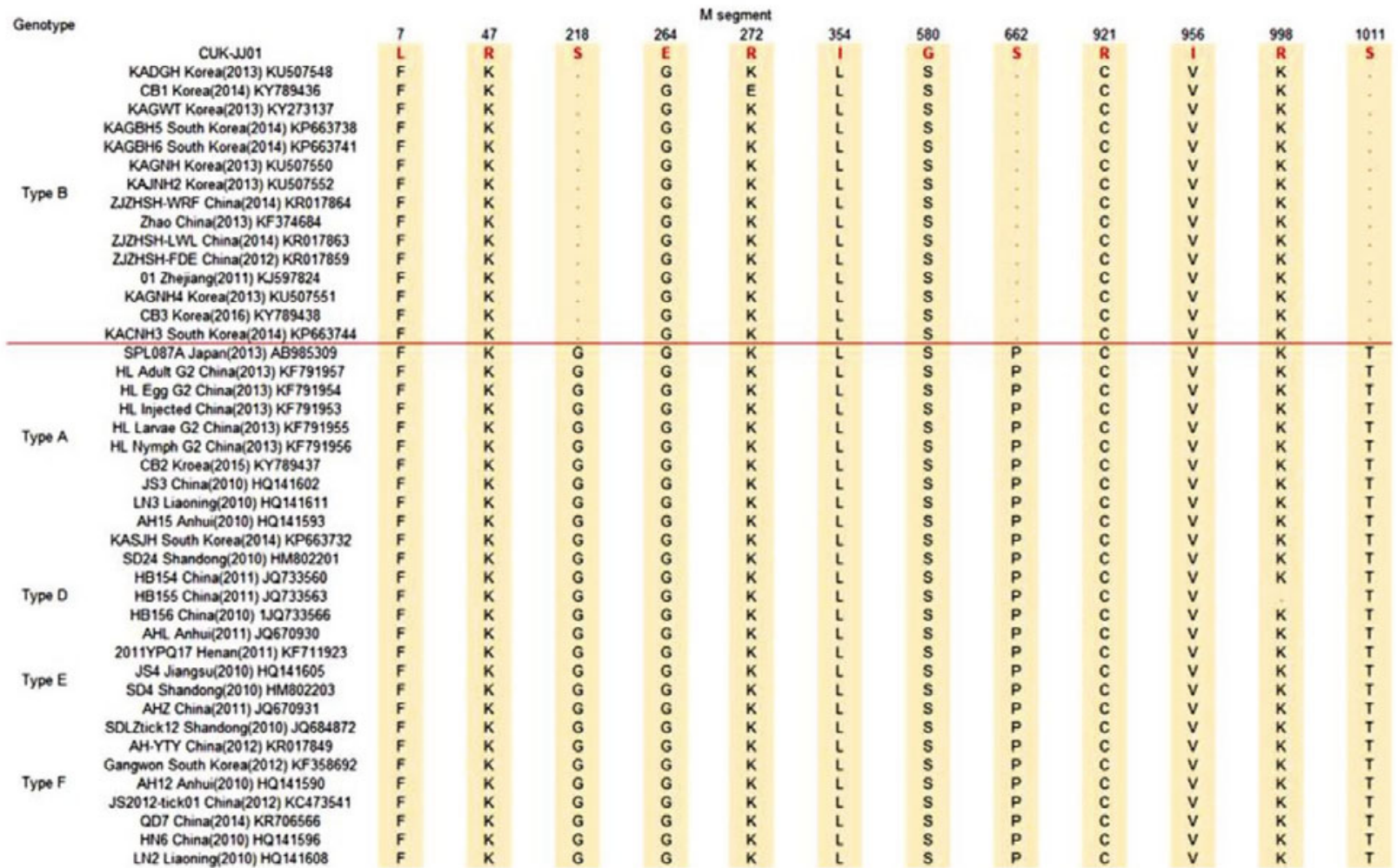

Fig. 1. Comparison of RNA-dependent RNA polymerase, glycoprotein/nucleoprotein, and nonstructural protein amino acid substitutions among SFTSV genotypes. Representative strains for each SFTSV genotype were selected. The red borders indicate the sites of amino acid changes. The portion marked in yellow indicates a location where a specific sequence change occurred for the specific genotype. SFTSV, severe fever with thrombocytopenia syndrome virus. 
Genotype

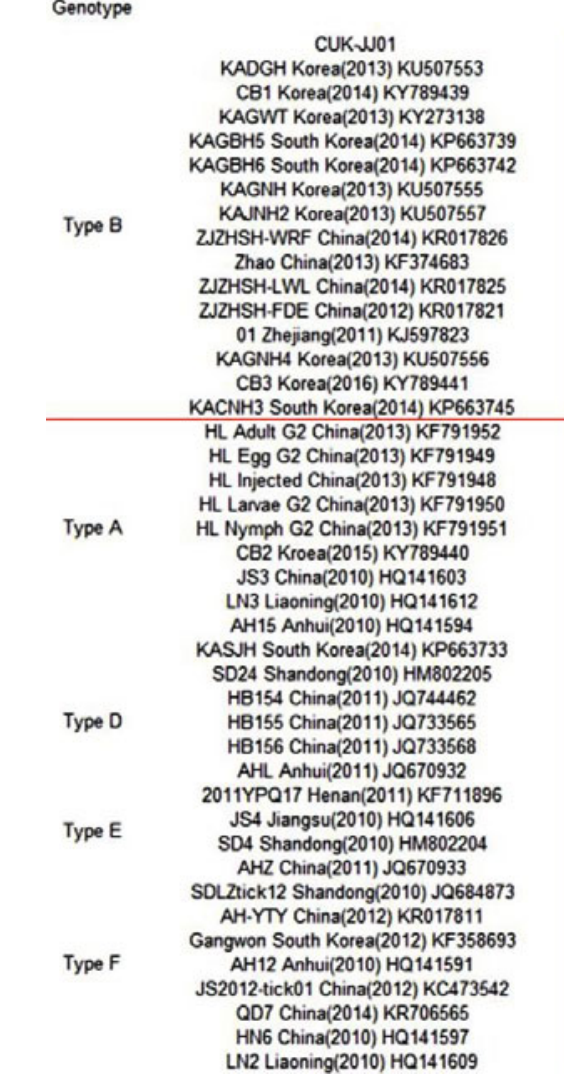

Genotype

S-Ns segment

$$
\text { CUKW01 }
$$

KADGH Korea(2013) KU507553 CB1 Korea(2014) KY789439 KAGWT Korea(2013) KY273138 KAGBH5 South Korea(2014) KP663739 KAGBH6 South Korea(2014) KP663742 KAGNH Korea(2013) KU507555 KAJNH2 Korea(2013) KU507557

$\begin{array}{ll}\text { Type B } & \text { KAJNH2 Korea(2013) KU507557 } \\ \text { ZJZHSH-WRF China(2014) KR017826 }\end{array}$ Zhao China(2013) KF374683
ZZHSH-WRF China(2014) KR0178 ZJZHSH-LWL China(2014) KR01782 ZJZHSH-FDE China(2012) KR017821 01 Zhejiang(2011) KJ597823 KAGNH4 Korea(2013) KU50755 CB3 Korea(2016) KY789441

KACNH3 South Korea(2014) KP66374 SPL087A Japan(2013) AB985541 HL Adult G2 China(2013) KF791952 HL Egg G2 China(2013) KF791949 HL Injected China(2013) KF791948 $\begin{array}{ll}\text { HL Lavae G2 China(2013) KF791950 } \\ \text { Type A } & \text { HL Nymph G2 China(2013) KF791951 }\end{array}$ CB2 Kroea(2015) KY789440 JS3 China(2010) HQ141603 LN3 Liaoning(2010) HQ141612 AH15 Anhui(2010) HQ141594

KASJH South Korea(2014) KP663733 SD24 Shandong(2010) HM802205 HB154 China(2011) JQ744462

$\begin{array}{ll} & \text { HB154 China(2011) JQ744462 } \\ \text { Type D } & \text { HB155 China(2011) JQ733565 } \\ & \text { HB156 China(2011) JQ733568 } \\ & \text { AHL Anhui(2011) JQ670932 }\end{array}$

$\begin{array}{ll}\text { Type D } & \text { HB155 China(2011) JQ733565 } \\ & \text { HB156 China(2011) JQ733568 } \\ & \text { AHL Anhui(2011) JQ670932 }\end{array}$

$\begin{array}{ll}\text { Type D } & \text { HB155 China(2011) JQ733565 } \\ & \text { HB156 China(2011) JQ733568 } \\ & \text { AHL Anhui(2011) JQ670932 }\end{array}$ 2011YPQ17 Henan(2011) KF711896

$\begin{array}{cc}\text { Type E } & \text { JS4 Jiangsu(2010) HQ141606 } \\ \text { SD4 Shandong(2010) HM802204 } \\ \text { AHZ China(2011) JQ670933 } \\ \text { SDLZtick12 Shandong(2010) JQ68487 }\end{array}$

$\begin{array}{cc}\text { Type E } & \text { JS4 Jiangsu(2010) HQ141606 } \\ \text { SD4 Shandong(2010) HM802204 } \\ \text { AHZ China(2011) JQ670933 } \\ \text { SDLZtick12 Shandong(2010) JQ68487 }\end{array}$ SDLZtick12 Shandong(2010) JQ6848
AH.YTY China(2012) KR017811

Gangwon South Korea(2012) KF358693

Type F AH12 Anhui(2010) HQ141591
JS2012-tick01 China(2012) KC473542 QD7 China(2014) KR706565 HN6 China(2010) HQ141597 LN2 Liaoning(2010) HQ141609
Fig. 1. Continued

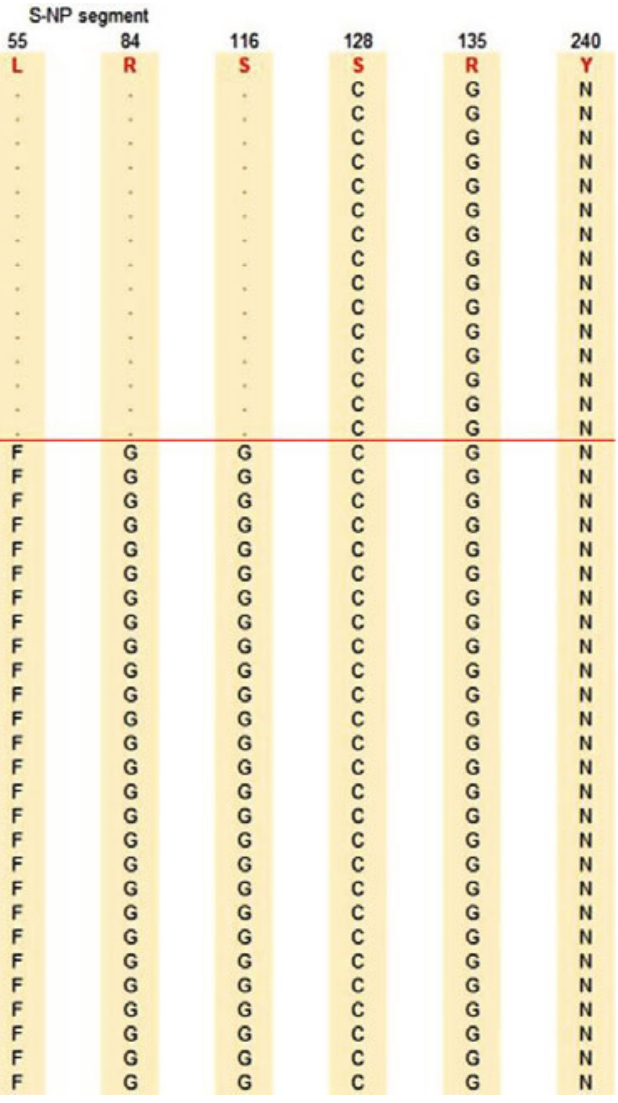

\begin{tabular}{|c|c|}
\hline 123 & 235 \\
\hline $\mathbf{N}$ & M \\
\hline S & L \\
\hline s & $\overrightarrow{\mathrm{L}}$ \\
\hline S & L \\
\hline $\mathrm{s}$ & $\mathrm{L}$ \\
\hline 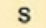 & L \\
\hline $\mathrm{s}$ & $\mathrm{L}$ \\
\hline S & L \\
\hline S & L \\
\hline $\mathrm{s}$ & L \\
\hline $\mathrm{s}$ & L \\
\hline $\mathrm{s}$ & L \\
\hline s & L \\
\hline $\mathrm{s}$ & L \\
\hline $\mathrm{s}$ & L \\
\hline$s$ & L \\
\hline $\mathrm{s}$ & $\mathrm{L}$ \\
\hline s & L \\
\hline $\mathbf{s}$ & L \\
\hline$s$ & L \\
\hline s & L \\
\hline$s$ & $\bar{L}$ \\
\hline s & $\bar{L}$ \\
\hline s & L \\
\hline s & $\mathrm{L}$ \\
\hline$s$ & L \\
\hline$s$ & L \\
\hline s & $\vec{L}$ \\
\hline$s$ & $\mathrm{~L}$ \\
\hline$s$ & $\vec{L}$ \\
\hline s & L \\
\hline s & $\overrightarrow{\mathrm{L}}$ \\
\hline 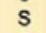 & $\mathrm{L}$ \\
\hline$s$ & $\mathrm{~L}$ \\
\hline s & L \\
\hline $\mathrm{s}$ & L \\
\hline $\mathrm{s}$ & L \\
\hline $\mathbf{s}$ & L \\
\hline $\mathrm{s}$ & L \\
\hline s & L \\
\hline $\mathrm{s}$ & L \\
\hline $\mathbf{s}$ & L \\
\hline $\mathrm{s}$ & L \\
\hline S & L \\
\hline
\end{tabular}




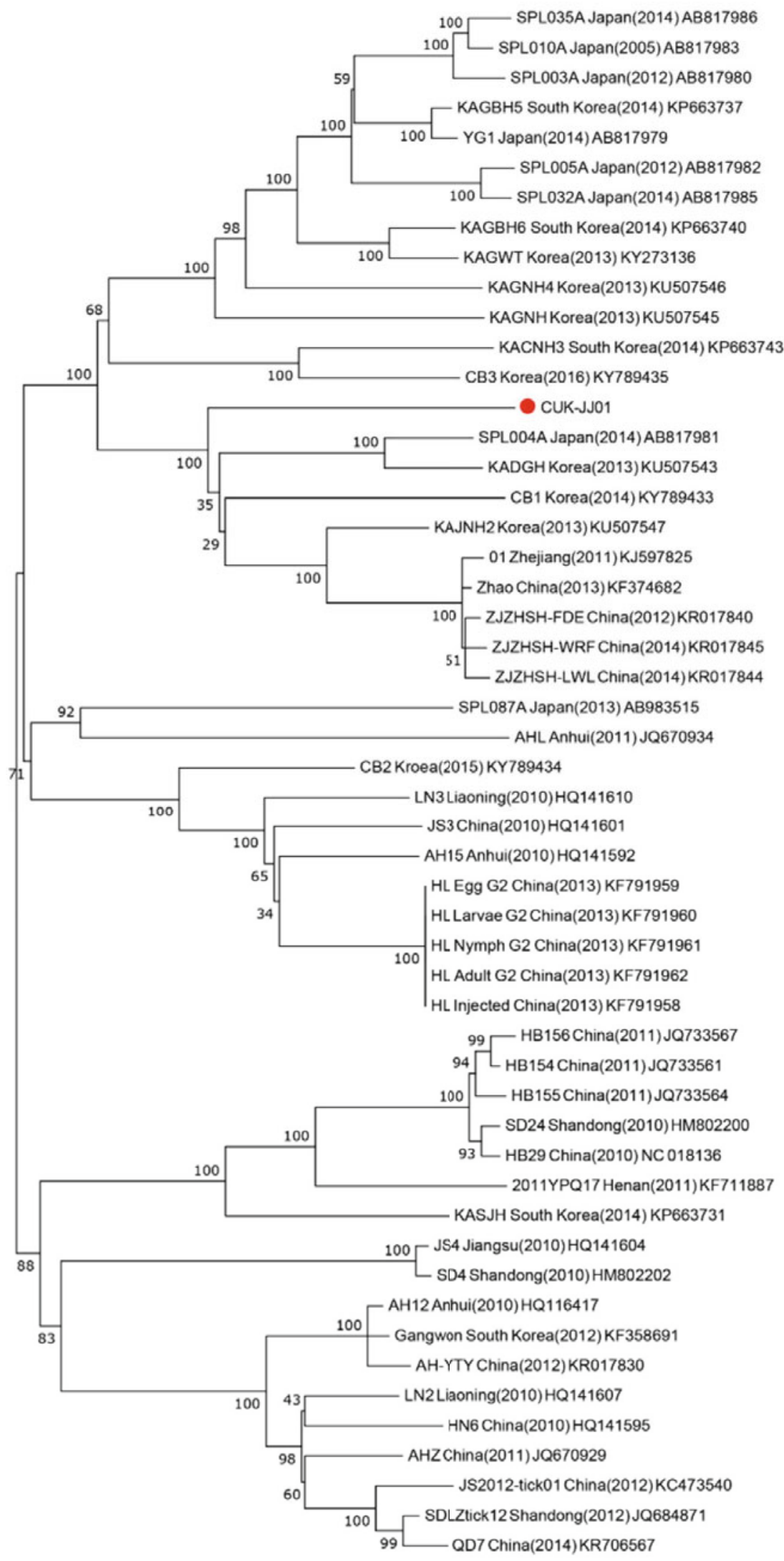

Fig. 2. Phylogenetic analysis using the complete nucleotide sequences of the L segments of SFTSV strains. We used an ML method based on the Kimura 2-parameter model, and the numbers on the branches indicate bootstrap percentages based on 1,000 replicates. The scale bar indicates the number of nucleotide substitutions per site. The phylogenetic branches were supported with greater than $70 \%$ bootstrap values. The human-derived South Korean SFTSV strains analyzed in this study are $B$ indicated by the red closed circles. ML, maximum likelihood; SFTSV, severe fever with thrombocytopenia syndrome virus.
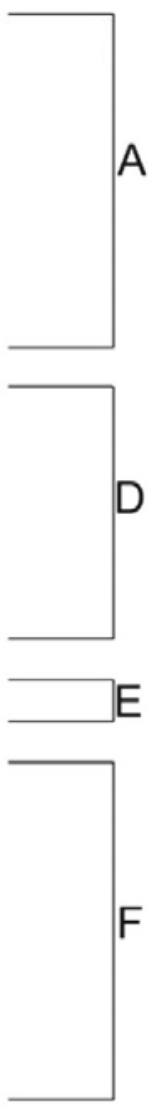


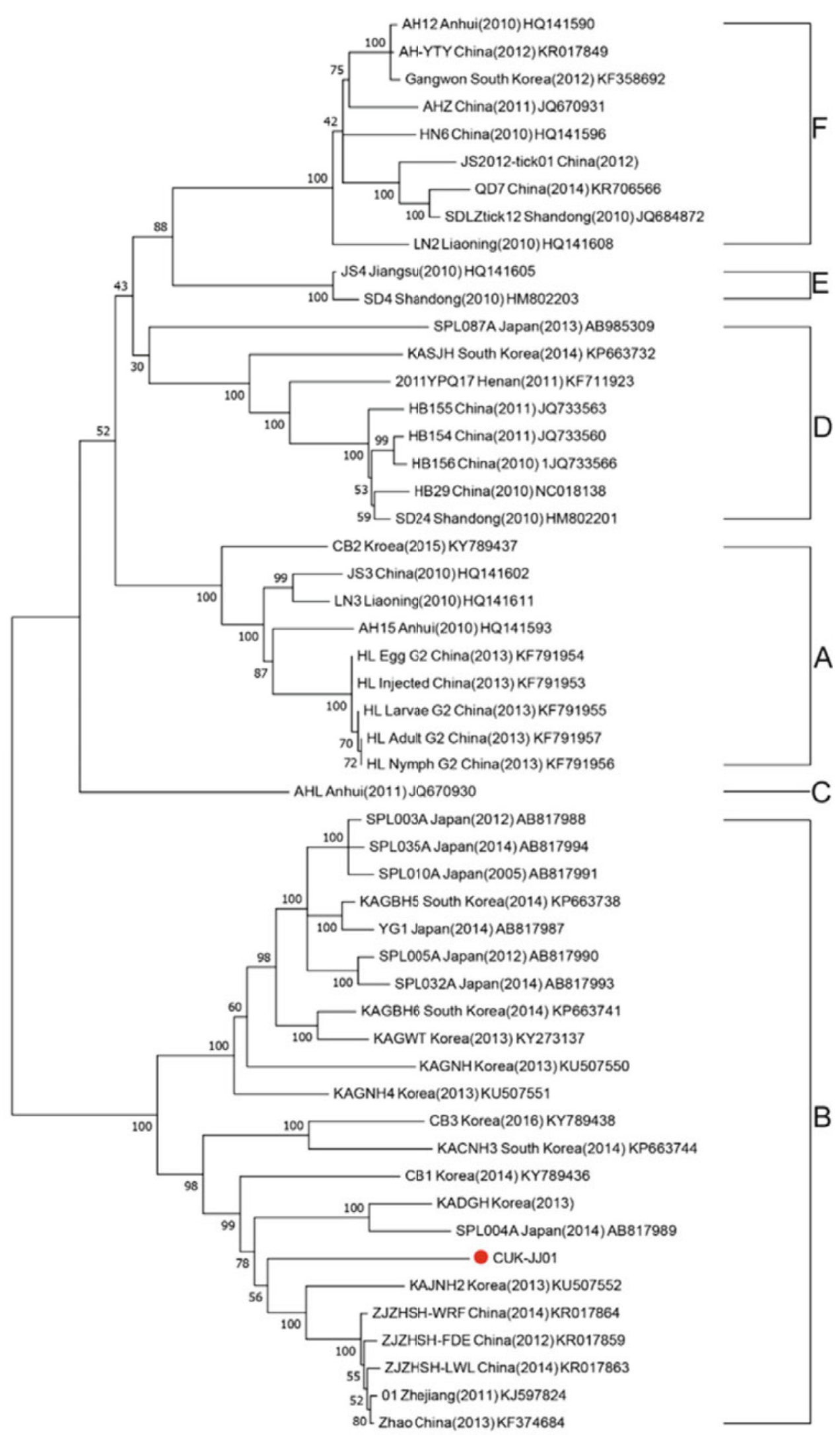

Fig. 3. Phylogenetic analysis using the complete nucleotide sequences of the M segments of SFTSV strains. We used an ML method based on the Kimura 2-parameter model, and the numbers on the branches indicate bootstrap percentages based on 1,000 replicates. The scale bar indicates the number of nucleotide substitutions per site. The phylogenetic branches were supported with greater than $70 \%$ bootstrap values. The human-derived South Korean SFTSV strains analyzed in this study are indicated by the red closed circles. ML, maximum likelihood; SFTSV, severe fever with thrombocytopenia syndrome virus.

B 


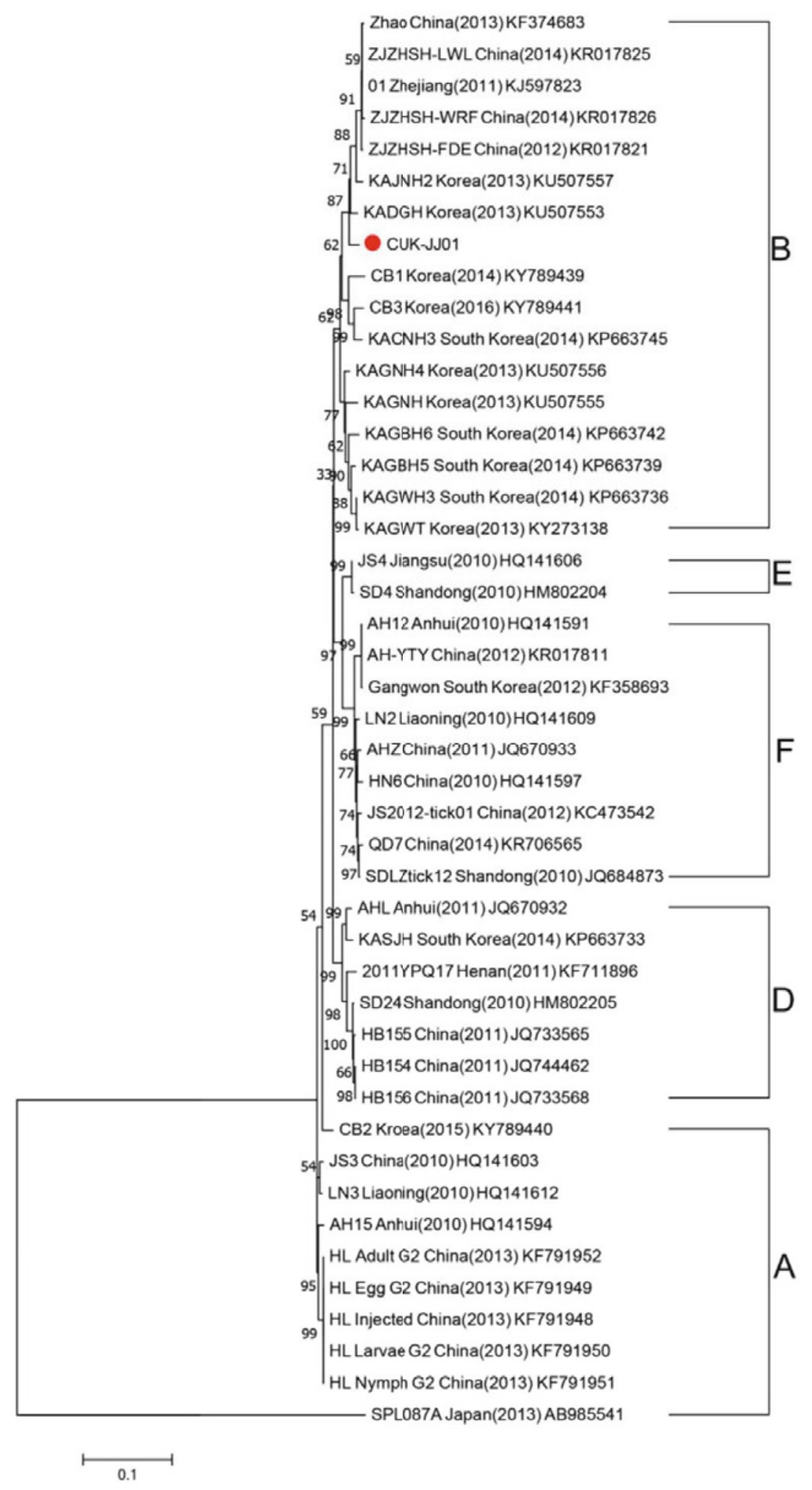

Fig. 4. Phylogenetic analysis using the complete nucleotide sequences of the S segments of SFTSV strains. We used an ML method based on the Kimura 2-parameter model, and the numbers on the branches indicate bootstrap percentages based on 1,000 replicates. The scale bar indicates the number of nucleotide substitutions per site. The phylogenetic branches were supported with greater than $70 \%$ bootstrap values. The human-derived South Korean SFTSV strains analyzed in this study are indicated by the red closed circles. ML, maximum likelihood; SFTSV, severe fever with thrombocytopenia syndrome virus.

being transitions and one (10\%) being a transversion; 12 of the $3222 \mathrm{nt}$ of the M segment displayed polymorphisms with $10(83 \%)$ being transitions and two (17\%) being transversion; and eight of the $1620 \mathrm{nt}$ of the $\mathrm{S}$ segment displayed polymorphisms with four (50\%) being transitions and four (50\%) nomes of 43 SFTSV strains, six of the $6255 \mathrm{nt}$ of the CUKJJ01 L segment displayed polymorphisms with five (90\%)

\section{Nucleotide and amino acid polymorphisms}

Based on sequence comparison of the three SFTSV RNA segments (L, M, and S) of CUK-JJ01 and the complete ge- 
being transversion (Fig. 1). Specifically, we identified three unique amino acid substitutions in the L segment of CUKJJ01 when compared to that of viruses from Haemaphysalis longicornis; the L segment of Haemaphysalis longicornis-derived viruses had Asp in aa 448, Ala in aa 449, and Glu in aa 586, while the L segment of CUK-JJ01 had Glu at aa 448, Thr at aa 449, and Asp at aa 586. These genotype-specific substitutions distinguished the different SFTSV genotypes. Among the 1074 aa of the M segment, three amino acid substitutions were detected in CUK-JJ01: aa 218 (Glu $\rightarrow$ Gly), aa 662 (Ser $\rightarrow$ Pro), and aa 1011 (Ser $\rightarrow$ Thr). Three amino acid substitutions were detected among the 540 aa of the $S$ segment of CUK-JJ01: aa 55 (Leu $\rightarrow$ Phe), aa 84 (Arg $\rightarrow$ Gly), and aa 116 (Ser $\rightarrow$ Gly).

\section{Phylogenetic analysis}

To investigate genetically the evolutionary origins and the relationship between the tick-derived and human-derived South Korean SFTSV strains, phylogenetic analyses were conducted using complete full-length sequences of CUK-JJ01 and previously isolated SFTSV strains from China, Japan, and South Korea. Phylogenetic analyses of the L, M, and S segments indicated that the tick-derived South Korean SFTSV strain KAGWT clustered with genotype B SFTSV strains that circulate in humans in China, Japan, and South Korea (Fig. 2). The CUK-JJ01 human isolate also clustered with genotype B viruses, although it was separated into a different sub-node than KAGWT and was closely related to a recent South Korean SFTSV human isolate KADGH, as well as the Chinese isolate ZJZHSH-FDE and Korean isolate KAJNH2. Overall, the phylogenetic trees revealed that South Korean SFTSV strains could be classified with high branch support into four distinct genotypes out of the six genotypes described previously (A, $\mathrm{B}, \mathrm{D}$, and F). No apparent genotypic differences were evident among the phylogenetic trees based on the SFTSV L, M, and $S$ segments and the complete genomes of 51 SFTSV strains (Figs. 2, 3, and 4). As previously reported, the L segment appeared to be the most highly conserved, whereas the S segment had the greatest number of nt polymorphisms. Moreover, the phylogenetic trees based on the S segment and the complete genome were nearly identical, which indicated that the $\mathrm{S}$ segment may be used to analyze the genetic relationships among SFTSV strains instead of the complete genome.

\section{Discussion}

SFTS was first recognized in 2007 and its causative agent SFTSV was identified in 2011 as a novel Phlebovirus (Xu et al., 2011; Yu et al., 2011; Zhang et al., 2011). SFTSV circulates in seven c central-eastern provinces of China and was more recently also found in South Korea, Japan, and Vietnam (Takahashi et al., 2014; Yun et al., 2014; Yoshikawa et al., 2015; Tran et al., 2019), which are separated from China by ocean straits. Recently, virus-bearing Amblyomma testudinarium ticks and migratory birds were shown to play a role in dispersing SFTSV to Vietnam (Tran et al., 2019). Thus, SFTSV poses a threat to public health due to its wide range of animal hosts and its ability to cause severe infections in humans, especially the elderly. Continuous monitoring and the mo- lecular detection of this virus are important for the correct diagnoses of SFTSV infections.

Since animals fail to show overt clinical symptoms upon infection and clinical symptoms of SFTSV infection in humans resemble those of other diseases, it is possible SFTSV is currently present in countries other than China, South Korea, Japan, and Vietnam. SFTSV genotypes A, D, and F are the most commonly detected genotypes in mainland China, while genotype B is most commonly found circulating in South Korea and Japan. Moreover, genotypes B, D, and F are all known to co-circulate in South Korea.

Due to the segmented nature of the SFTSV genome, genetic reassortments have occurred in strains from China and Japan, resulting in at least ten strains with unique genotypes (Fu et al., 2016; Shi et al., 2017). In the current study, the genome sequence of a virus isolated from a patient who died from SFTS in Jeju Island in October 2017 was analyzed and compared to the whole-genome sequences of other human-derived strains. The analysis revealed that consistent with all SFTSV strains, CUK-JJ01 consisted of three genomic segments, including a 6368-nt L segment, a 3378-nt M segment, and a 1744-nt S segment. Furthermore, CUK-JJ01 belongs to genotype B and showed no evidence of intra- or inter-genogroup recombination of the $\mathrm{L}, \mathrm{M}$, or S segment. Sequence analysis of CUK-JJ01 revealed that the L segment was $97.49 \%$ similar to that of KAJNH2, the M segment was 97.18\% similar to that of ZJZHSH-FDE, and the S segment was $97.94 \%$ similar to that of KADGH. Genetic and phylogenetic analyses of full-length genome sequences revealed that tick-derived and human-derived South Korean SFTSV strains are closely related and that at least four different genotypes of SFTSV are co-circulating in South Korea.

To date, only few studies have been conducted on SFTSV in Korean population, and most are limited to serological analysis. In China and Japan, the number of patients with SFTSV is increasing, and various genotypes have been detected since 2010. In particular, new SFTSV genotypes, reassortments, and recombination isolates have been identified by long-term molecular epidemiological analysis.

Although the present study has limitations in terms of not analyzing multiple samples, the findings should serve as basis for the development of methods for diagnosis and to detect viral recombinants, as well as to elucidate the transmission and spread of SFTSV by comparing the whole genome sequence of SFTSV isolated in South Korea and that of foreign isolates. In the future, it is anticipated that gene and mutation analyses using animal-derived viruses can shed light into inter-species propagation and emergence of new viruses.

Although there have been several studies focusing on the pathogenesis and transmission of SFTSV, there is currently no vaccine available to prevent its spread. Initial studies indicate a higher mortality rate in patients 50 years of age and above, suggesting that susceptibility to SFTSV infection may vary and be dependent upon several risk factors, including the age and immune status of the patient (Robles et al., 2018). Nonetheless, with initial indications of overall mortality rates from $30-40 \%$ in China, South Korea, and Japan, and a wide range of host species that have direct human contact but display limited clinical illnesses, vaccine development will be critical for the prevention of SFTS and any hope for the 
eradication of SFTSV.

The results from the current study provide insight into the genetic origins of human of SFTSV strains, as well as shed light on the molecular epidemiology, genetic diversity, and evolution of SFTSV. These associations have important implications in the design and development of diagnostic procedures and vaccines against SFTSV. Moreover, the information acquired from the whole-genome sequence of SFTSV strain CUK-JJ01 may prove useful for obtaining more accurate diagnoses of SFTSV and for advancing research toward the elucidation of genetic functions, the prediction of newly appearing pandemic variants via comparison with SFTSVs in neighboring countries, and in vaccine development. Overall, broadening the information base and genetic resources regarding SFTSVs circulating globally will provide important benefits for public health and help to identify new emerging strains of SFTSV.

\section{Acknowledgements}

This work was supported by a grant from the Korea Health Technology R\&D Project through the Korea Health Industry Development Institute (KHIDI) funded by the Ministry of Health \& Welfare, Republic of Korea (grant number HI15C1781); and the Korea Ministry of Environment (MOE) as a Public Technology Program based on Environmental Policy (grant number 2016000210002).

\section{Conflicts of Interest}

The authors declare no conflicts of interest.

\section{References}

Bao, C.J., Guo, X.L., Qi, X., Hu, J.L., Zhou, M.H., Varma, J.K., Cui, L.B., Yang, H.T., Jiao, Y.J., Klena, J.D., et al. 2011. A family cluster of infections by a newly recognized bunyavirus in Eastern China, 2007: further evidence of person-to-person transmission. Clin. Infect. Dis. 53, 1208-1214.

Ding, F., Zhang, W., Wang, L., Hu, W., Soares Magalhaes, R.J., Sun, H., Zhou, H., Sha, S., Li, S., Liu, Q., et al. 2013. Epidemiologic features of severe fever with thrombocytopenia syndrome in China, 2011-2012. Clin. Infect. Dis. 56, 1682-1683.

Fu, Y., Li, S., Zhang, Z., Man, S., Li, X., Zhang, W., Zhang, C., and Cheng, X. 2016. Phylogeographic analysis of severe fever with thrombocytopenia syndrome virus from Zhoushan Islands, China: implication for transmission across the ocean. Sci. Rep. 6, 19563.

Kim, K.H., Yi, J., Kim, G., Choi, S.J., Jun, K.I., Kim, N.H., Choe, P.G., Kim, N.J., Lee, J.K., and Oh, M.D. 2013. Severe fever with thrombocytopenia syndrome, South Korea, 2012. Emerg. Infect. Dis. 19, 1892-1894.

Korea Center for Disease Control (KCDC). 2018. Disease web statistics system.

Liu, K., Cui, N., Fang, L.Q., Wang, B.J., Lu, Q.B., Peng, W., Li, H., Wang, L.Y., Liang, S., Wang, H.Y., et al. 2014a. Epidemiologic features and environmental risk factors of severe fever with thrombocytopenia syndrome, Xinyang, China. PLoS Negl. Trop. Dis. 8 , e2820.

Liu, Q., He, B., Huang, S.Y., Wei, F., and Zhu, X.Q. 2014b. Severe fever with thrombocytopenia syndrome, an emerging tick-borne zoonosis. Lancet Infect. Dis. 14, 763-772.

Niu, G., Li, J., Liang, M., Jiang, X., Jiang, M., Yin, H., Wang, Z., Li, C., Zhang, Q., Jin, C., et al. 2013. Severe fever with thrombocytopenia syndrome virus among domesticated animals, China. Emerg. Infect. Dis. 19, 756-763.

Park, S.W., Han, M.G., Yun, S.M., Park, C., Lee, W.J., and Ryou, J. 2014. Severe fever with thrombocytopenia syndrome virus, South Korea, 2013. Emerg. Infect. Dis. 20, 1880-1882.

Robles, N.J.C., Han, H.J., Park, S.J., and Choi, Y.K. 2018. Epidemiology of severe fever and thrombocytopenia syndrome virus infection and the need for therapeutics for the prevention. Clin. Exp. Vaccine Res. 7, 43-50.

Saitou, N. and Nei, M. 1987. The neighbor-joining method: a new method for reconstructing phylogenetic trees. Mol. Biol. Evol. 4, 406-425.

Shi, J., Hu, S., Liu, X., Yang, J., Liu, D., Wu, L., Wang, H., Hu, Z., Deng, F., and Shen, S. 2017. Migration, recombination, and reassortment are involved in the evolution of severe fever with thrombocytopenia syndrome bunyavirus. Infect. Genet. Evol. 47, 109-117.

Takahashi, T., Maeda, K., Suzuki, T., Ishido, A., Shigeoka, T., Tominaga, T., Kamei, T., Honda, M., Ninomiya, D., Sakai, T., et al. 2014. The first identification and retrospective study of severe fever with thrombocytopenia syndrome in Japan. J. Infect. Dis. 209, 816-827.

Tamura, K., Stecher, G., Peterson, D., Filipski, A., and Kumar, S. 2013. MEGA6: Molecular evolutionary genetics analysis version 6.0. Mol. Biol. Evol. 30, 2725-2729.

Tran, X.C., Yun, Y., Van An, L., Kim, S.H., Thao, N.T.P., Man, P.K.C., Yoo, J.R., Heo, S.T., Cho, N.H., and Lee, K.H. 2019. Endemic severe fever with thrombocytopenia syndrome, Vietnam. Emerg. Infect. Dis. 25, 1029-1031.

Xu, B., Liu, L., Huang, X., Ma, H., Zhang, Y., Du, Y., Wang, P., Tang, X., Wang, H., Kang, K., et al. 2011. Metagenomic analysis of fever, thrombocytopenia and leukopenia syndrome (FTLS) in Henan Province, China: discovery of a new bunyavirus. PLoS Pathog. 7, e1002369.

Yoshikawa, T., Shimojima, M., Fukushi, S., Tani, H., Fukuma, A., Taniguchi, S., Singh, H., Suda, Y., Shirabe, K., Toda, S., et al. 2015. Phylogenetic and geographic relationships of severe fever with thrombocytopenia syndrome virus in China, South Korea, and Japan. J. Infect. Dis. 212, 889-898.

Yu, X.J., Liang, M.F., Zhang, S.Y., Liu, Y., Li, J.D., Sun, Y.L., Zhang, L., Zhang, Q.F., Popov, V.L., Li, C., et al. 2011. Fever with thrombocytopenia associated with a novel bunyavirus in China. $N$. Engl. J. Med. 364, 1523-1532.

Yun, Y., Heo, S.T., Kim, G., Hewson, R., Kim, H., Park, D., Cho, N.H., Oh, W.S., Ryu, S.Y., Kwon, K.T., et al. 2015. Phylogenetic analysis of severe fever with thrombocytopenia syndrome virus in South Korea and migratory bird routes between China, South Korea, and Japan. Am. J. Trop. Med. Hyg. 93, 468-474.

Yun, S.M., Lee, W.G., Ryou, J., Yang, S.C., Park, S.W., Roh, J.Y., Lee, Y.J., Park, C., and Han, M.G. 2014. Severe fever with thrombocytopenia syndrome virus in ticks collected from humans, South Korea, 2013. Emerg. Infect. Dis. 20, 1358-1361.

Zhang, Y.Z., Zhou, D.J., Qin, X.C., Tian, J.H., Xiong, Y., Wang, J.B., Chen, X.P., Gao, D.Y., He, Y.W., Jin, D., et al. 2012. The ecology, genetic diversity, and phylogeny of Huaiyangshan virus in China. J. Virol. 86, 2864-2868.

Zhang, Y.Z., Zhou, D.J., Xiong, Y., Chen, X.P., He, Y.W., Sun, Q., Yu, B., Li, J., Dai, Y.A., Tian, J.H., et al. 2011. Hemorrhagic fever caused by a novel tick-borne bunyavirus in Huaiyangshan, China. Zhonghua Liu Xing Bing Xue Za Zhi 32, 209-220.

Zhao, L., Zhai, S., Wen, H., Cui, F., Chi, Y., Wang, L., Xue, F., Wang, Q., Wang, Z., Zhang, S., et al. 2012. Severe fever with thrombocytopenia syndrome virus, Shandong province, China. Emerg. Infect. Dis. 18, 963-965. 